\title{
Review
}

\section{The Case for Digital Pill Use in Clinical Trials}

\author{
Gerald L. Klein, MD* \\ MedSurgPI LLC, 3700 Lark Farm Road, Franklinton, NC 27525, USA \\ "Corresponding author \\ Gerald L. Klein, MD \\ Principal, MedSurgPI LLC, 3700 Lark Farm Road, Franklinton, NC 27525, USA;Tel. 9|9-930-9|80; E-mail: gklein@medsurgpi.com
}

\section{Article information}

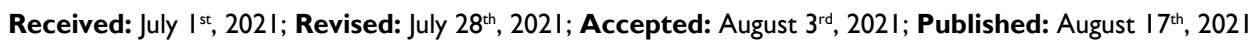

\section{Cite this article}

Klein GL. The case for digital pill use in clinical trials. Clin Trial Pract Open J. 202I; 4(I): 16-2I. doi: I0.17।40/CTPOJ-4-I20

\section{ABSTRACT}

Medication adherence in clinical trials is significantly overestimated through every phase of drug development. This can cause a reduction in statistical power, potentially resulting in incorrect conclusions regarding efficacy, safety, tolerability, and dose-response relationships, in addition to major cost overruns. Digital pill systems enable adherence measurement through an embedded ingestible sensor paired with an external receiver. An oral pharmaceutical product is over-encapsulated by a pharmaceutical-grade shell containing a biocompatible sensor. Upon exposure to gastrointestinal fluid, the shell dissolves and the sensor is activated. Medication ingestion data is transmitted via a digital signal. Clinicians and researchers use this data to track, in real time, when and if a medication was taken. These systems have demonstrated a 99.4\% rate of accuracy, and have over 15-years of supporting experience and safety data. Spurred by the accelerated adoption of technology in healthcare and in everyday life, patients have become tech-savvy. They quickly adapt to these devices, and are able to use them safely and effectively. Digital pills can be implemented in most types of studies. In early-stage trials such as pharmacokinetic and pharmacodynamic studies, or dose-finding studies, accurate information on maximum-tolerated dose levels is essential and cannot be established unless study participants are highly adherent. In later-stage pivotal trials, effective medication adherence tracking can strengthen the dataset and confidence in the study results. Significant nonadherence may generate results that do not meet statistical or clinical significance for the critical endpoints, resulting in at worst, a failed trial, or at best, the need to enroll additional patients at substantial additional cost. Most clinical trials fail to achieve statistical significance, and poor medication adherence is often an important contributor. A digital pill system can ensure the quality and integrity of adherence data, increase confidence in the overall study data, and improve clinical trial efficiency.

\section{Keywords}

Digital pills; Digital medication; Ingestible event marker; Medication adherence; Medication nonadherence;

Remote patient monitoring; Clinical trials; Drug development; ID-Cap System; Data integrity.
\end{abstract}

\section{INTRODUCTION}

$\mathrm{T}$ hose who design and run clinical trials often worry about medication adherence among trial participants. Nonadherence is a significant concern in all types of clinical trials, from earlier stages of drug development (including pharmacokinetic, pharmacodynamic, dose-finding, and dose optimization studies) to latestage pivotal trials and post-marketing studies. Medication adherence is often neglected at every phase of drug development and in clinical research. This can and has led to reduced statistical power and incorrect conclusions regarding efficacy and dose-response relationships. ${ }^{1-6}$

This paper explores the misconception of near-perfect adherence in clinical trials, and the possible consequences of non- adherence. Plus we will explore a new technology digital pill systems-that allows confirmation of medication ingestion at a dose level.

\section{THE IMPACT OF NONADHERENCE ON CLINICAL TRIALS}

Medication adherence in clinical trials is dramatically overestimated. ${ }^{1}$ A review of data from 95 clinical studies with a total of 16,907 participants, in studies ranging from 30 to 1,400 days, found a steady decrease in medication persistence over time. There was an immediate $4 \%$ drop simply from noninitiation of the study medication. At day 100 , only $80 \%$ of the remaining patients were still following the dosing regimen. After one year, a full $40 \%$ of participants had stopped taking their medications. ${ }^{2}$ 
Lack of persistence is one component of nonadherence and accounts for the largest share of patient drop-off in clinical studies. Another facet of nonadherence is improper execution, which may manifest as deviations from the prescribed dosing regimen or interruptions in therapy due to a variety of factors including, memory lapses, gaps in understanding, drug holidays, adverse events, etc. ${ }^{2}$ These frequently occur in clinical studies, just as they do in clinical practice. In the same study referenced above, on day $100,12 \%$ of patients who qualified as persistent failed to take their medication as instructed. ${ }^{2}$ These failures of execution impact treatment effectiveness and may further contribute to permanent discontinuation of the study drug by the patient. Taken together, lack of persistence and nonexecution of the dosing regimen can negatively affect study results by increasing data variability. ${ }^{2}$

\section{THE OUTSIZED INFLUENCE OF ADHERENCE ON CLINICAL TRIAL COSTS}

The cost of enrolling patients is an important (and expensive) line item in clinical trial operational budgets. In a 2014 survey, per-patient enrollment costs were estimated at $\$ 15,700$ in Phase 1 clinical studies and $\$ 26,000$ in Phase 3 and 4 clinical studies. ${ }^{7}$

In a 2017 publication, author Moe Alsumidaie estimates that $56 \%$ more patients would be needed in a clinical trial to maintain statistical power with a $40 \%$ nonadherence rate, and presents the costs of enrolling the additional patients needed. He also estimates the savings realized for each $1 \%$ improvement in adherence (Table 1). ${ }^{7}$ It is striking to note the savings, especially in Phase 3 and 4 trials, with such a small percentage improvement in adherence.

Certainly, adherence missteps can increase the risk of type I and type II errors, and can negatively impact understanding of both the efficacy and the toxicity of a given therapeutic. ${ }^{1-6}$ In the process of clinical drug development, unnecessary delays and stalled programs are expensive mistakes for a sponsor. Yes, there are the operational costs associated with timeline slippage, but there is also the opportunity cost associated with lost market revenue, which can be millions of dollars per day in the case of blockbuster drugs. ${ }^{3}$

Digital pills are a solution to the nonadherence predicament. There is a wealth of evidence supporting use of digital pills in clinical trials, and recent advances in device reliability and patient acceptability maximize the benefits of tracking adherence while simultaneously easing the implementation effort. The quality and integrity of accurate adherence data and reduction in data variability will pay dividends in the long run.

\section{WHAT IS A "DIGITAL PILL”?}

Digital pill systems are ingestible event markers that enable adherence measurement through an embedded ingestible sensor paired with an external receiver. Oral medications are over-encapsulated by a pharmaceutical-grade capsule shell that contains the biocompatible sensor; upon entering the stomach, the capsule shell dissolves and the sensor is activated by exposure to the gastrointestinal fluid. A digital signal transmits medication ingestion data in real time. ${ }^{8,9} \mathrm{~A}$ wearable device picks up the signal and transmits the information for use by the clinician and researcher.

The process of implementing a digital pill is identical to the process of over-encapsulating an active study drug or a placebo product for blinding purposes, except that the capsule also contains an ingestible sensor. In bioequivalence studies and dissolution tests, digital pills were found to be equivalent to native drug formulations and met United States Pharmacopeia (USP) guidelines for dissolution. ${ }^{8}$ Digital pills have also shown long-term stability when stored in real-world conditions during long-term use. ${ }^{9}$

Fifteen-years of experience and safety data support the use of digital pills, with over 140,000 ingestions recorded in more than 1,000 patients who have used these devices safely and effectively. ${ }^{10}$ Digital pill systems have demonstrated a high rate of accuracy, with one study showing $99.4 \%$ adherence $(2,807$ ingestions detected/2,824 ingestions prescribed) in kidney transplant patients. ${ }^{11}$

Patients can quickly learn to use such devices and readily accept them. In a recent usability study, patient users were able to effectively and safely use the system as intended; in qualitative assessments, they concluded that the device was easy to use and had potential to be a valuable tool for helping to manage their medications. ${ }^{12}$ In a study examining opioid use patterns, a $90 \%$ positive experience using a digital pill system was reported. ${ }^{13}$

\section{WHAT TYPE OF DIGITAL PILL SYSTEMS ARE AVAILABLE NOW?}

There are two digital pill systems that have been cleared for marketing by the Food and Drug Administration (FDA) as ingestible event markers. Otsuka Pharmaceutical Co., Ltd. (Tokyo, Japan) collaborated with Proteus Digital Health on the development and

\begin{tabular}{|c|c|c|c|c|c|}
\hline $\begin{array}{l}\text { Trial } \\
\text { Phase }\end{array}$ & $\begin{array}{c}\text { Average \# Patients per } \\
\text { Trial, 2014 }\end{array}$ & $\begin{array}{l}\text { Average Cost of } \\
\text { Enrolling One } \\
\text { Patient }\end{array}$ & $\begin{array}{l}\text { Estimated Average Additional Patients } \\
\text { Needed to Maintain Statistical Power } \\
\text { at } \mathbf{4 0 \%} \text { Nonadherence }\end{array}$ & $\begin{array}{l}\text { Estimated Cost of Enrolling } \\
\text { Additional Patients Needed } \\
\text { due to } 40 \% \text { Nonadherence* }\end{array}$ & $\begin{array}{c}\text { Average Savings per } \\
\text { I\% Improvement in } \\
\text { Adherence* }\end{array}$ \\
\hline Phase I & 75 & $\$ 15,700$ & 42 & $\$ 655,475$ & $\$ 19,036$ \\
\hline Phase 2 & 203 & $\$ 19,300$ & 113 & $\$ 2,171,250$ & $\$ 50,904$ \\
\hline Phase 3 & 828 & $\$ 26,000$ & 460 & $\$ 11,960,000$ & $\$ 335,725$ \\
\hline Phase 4 & 422 & $\$ 26,000$ & 234 & $\$ 6,093,750$ & $\$ 167,050$ \\
\hline
\end{tabular}


marketing of the first adherence-enabled drug-device combination product in Abilify MyCite ${ }^{\circledR}$ (Otsuka subsequently acquired the Proteus technology). ${ }^{14,15}$

The ID-Cap ${ }^{\mathrm{TM}}$ System from etectRx (Gainesville, FL, USA) was granted $510(\mathrm{k})$ clearance by the FDA in December of 2019. This system is a platform technology that can easily encapsulate a wide range of oral therapeutic agents. The components of the ID-Cap System, and a schematic of how the system works, are shown in Figures 1 (a-d) and 2.

\section{HOW CAN DIGITAL PILLS BE IMPLEMENTED IN CLINICAL TRIALS?}

Some readers may be wondering: why should I use a digital pill as opposed to other methods of tracking adherence? Will a digital pill be difficult to implement? What type of study is the best for use

Figure Ia. ID-Capsule: A Digital Pill Consisting of a Pharmaceutical-Grade Capsule Shell with an Embedded Ingestible Sensor

The ID-Capsule has been designed to encapsulate a wide range of oral medications. The sensor communicates a digital signal shortly following ingestion and capsule dissolution. The sensor is naturally and safely eliminated through the patient's gastrointestinal (GI) tract.

Figure Ib. Reader: A Wearable Device that Detects Messages Transmitted from the Ingested Sensor and Forwards them to the Patient App and Clinician Dashboard

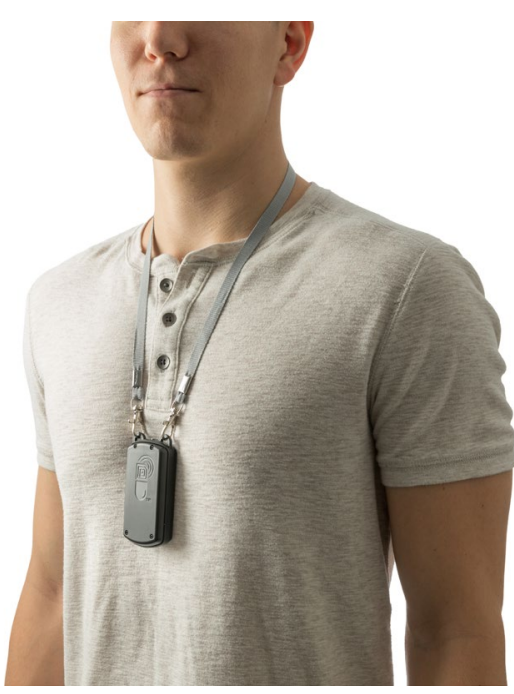

of a digital pill?

There are other ways of tracking medication adherence in clinical research, including patient self-reports, adherence-reporting mobile applications, pill counts, pharmacy dispensing data, electronic pill dispensers, and other solutions, but none have been optimized and many are not reliable. ${ }^{3,16}$ Despite widespread collection of data using these methods in clinical studies, the utility of such data is hampered by the accuracy and integrity of the information and completeness of the dataset.,16 Digital pills have proven accuracy and reliability, and are well-accepted by patients. ${ }^{10-14}$

Implementation of a digital pill system is a streamlined process and will not require deviation from already established work patterns or behaviors within clinical operations. Many researchers currently over-encapsulate oral medications for controlled trials; co-encapsulating medications with an ingestible sen-

Figure Ic. Patient App:Allows Patients to View Ingestion Events in Real Time as well as their Medication Use History.The App can also Send Patient Reminders and Alerts

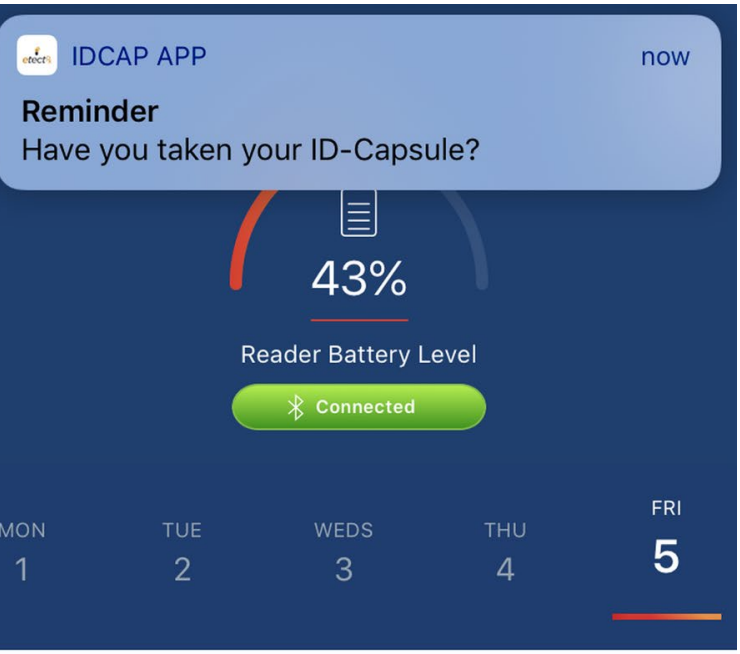

Jul 05, 9:49 AM $\diamond$ ID-Capsule

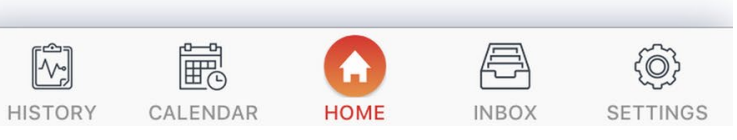



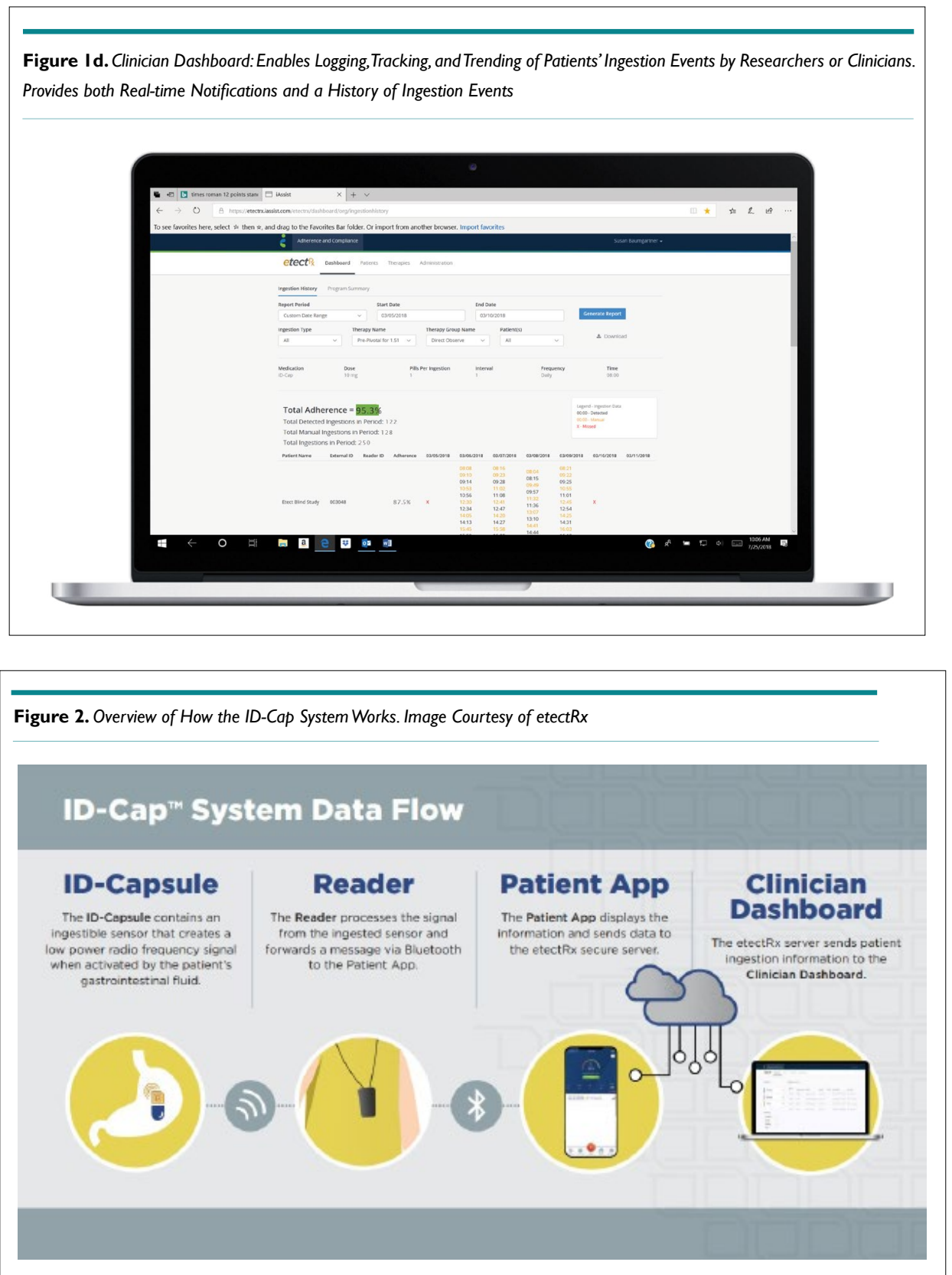

sor will be a familiar process. Patients are sophisticated users of digital devices and wearables, and will engage with and adapt to the technology easily.

As for the question of what type of clinical studies are best for digital pill use, the answer is really any type of study. In early-stage human trials, such as pharmacokinetic, pharmacodynamic, or dose-finding studies, the need for highly adherent study participants is critical. ${ }^{17}$ If these results do not reflect accurate information, efficacious, safe, and well-tolerated dosing cannot be established, and drug development may be adversely impacted.

In later-stage pivotal trials, it is of the utmost importance that medication regimens are strictly followed and that measured adherence data are incorporated into the data analyses. Especially in intent-to-treat (IT'T) analyses, where outcomes depend on treatment effect but also protocol adherence, effective medication ad- herence tracking can strengthen the dataset and confidence in the study results. ${ }^{18}$

\section{BEST PRACTICES IN CLINICALTRIAL DIGITAL PILL USE}

A rigorous approach to measuring medication adherence and evaluation of electronic monitoring of medication intake should be evaluated for every drug development program.

There are many scenarios with especially pressing needs for digital pill implementation:

- Any trial requiring the measurement of medication adherence (in fact, there are efforts underway to develop protocol design guidance for adherence measurement and reporting in trials). ${ }^{3}$ 
- Studies where the intervention is a self-administered oral therapeutic.

- Comparative studies assessing non-inferiority, where proving differentiation is important or differences may be small.

- Decentralized or virtual trials with requirements for remote patient monitoring, including those conducted during a pandemic.

- When drug accountability is critical (for example, in cases where there is limited supply of an active pharmaceutical ingredient, when diversion potential is high, or with high-cost medications).

- Conditions in which nonadherence presents a significant clinical risk to the patient, or in which adherence is known to be suboptimal, such as:

o Oncology:

- Complicated, non-recurring dosing schedules make adherence difficult for the patient, and the risks of remission with nonadherence are high. ${ }^{19-21}$

- In a study of molecular responses to treatment for chronic myeloid leukemia, adherence to imatinib (measured by an electronic medication monitoring system) was associated with molecular response. No complete molecular responses were observed when adherence was $<90 \%$, and no major molecular responses were observed when adherence was $<80 \%{ }^{22}$

o Neurology:

- Disease progression in multiple sclerosis or Parkinson's disease, for example.

- Therapeutic agents in this category often have complicated dosing schedules, plus there is a critical need to keep drug levels within the therapeutic range. Treatment adherence to oral disease-modifying agents for multiple sclerosis is estimated to be only $72.6 \%$ in the USA..$^{23}$

o Infectious Disease:

- Viral suppression of human immunodeficiency virus (HIV), HIV treatment, HIV prevention with pre-exposure prophylaxis (PrEP), cure with Hepatitis C therapy.

- Digital pill systems have been used with success in HIV and Hepatitis C, disease states where medication adherence has been problematic in the past and is essential for successful therapeutic outcomes. $^{24}$

Digital pill systems record and report objective medication adherence data in real time and facilitate early identification of episodes and patterns of nonadherence and suboptimal adherence. Remote patient monitoring of self-administered medications by clinical researchers in real time enables insight into the real-world context of medication use and delivery of evidence-based clinical and behavioral interventions for improvement of adherence, persistence, and therapeutic outcomes. The study participant can be engaged at the moment of nonadherence, allowing personalized care, patient activation strategies, and technology-based interventions that support the study protocol.

\section{CONCLUSION}

Most clinical trials fail to achieve statistical significance, ${ }^{25}$ and, in many instances, poor medication adherence is to blame, a silent predator negatively impacting study results. ${ }^{2}$ An ingestible event marker or digital pill system that accurately measures adherence could make the difference between a successful clinical trial and a failed study.

Ensuring the quality and integrity of adherence data and its impact on overall study data will pay dividends in decreased drug development timelines and costs, and increased clinical trial success and efficiency. Digital pills are an innovative solution to the age-old problem of medication adherence; with a streamlined implementation process and positive track record of patient acceptance, they are worth a look for your next clinical trial.

\section{ACKNOWLEDGEMENTS}

The author would like to thank Kate Jones of Global MedCom Consulting, LLC and Susan Baumgartner, PharmD, MBA of etectRx, Inc. for their editorial assistance in the preparation of this manuscript.

\section{REFERENCES}

1. Vrijens B, Urquhart J. Methods of measuring, enhancing, and accounting for medication adherence in clinical trials. Clin Pharmacol Ther. 2014; 95(6): 617-626. doi: 10.1038/clpt.2014.59

2. Blaschke TF, Osterberg L, Vrijens B, Urquhart J. Adherence to medications: insights arising from studies on the unreliable link between prescribed and actual drug dosing histories. Annu Rev Pharmacol Toxicol. 2012; 52: 275-301. doi: 10.1146/annurev-pharmtox-011711-113247

3. Eliasson L, Clifford S, Mulick A, Jackson C, Vrijens B. How the EMERGE guideline on medication adherence can improve the quality of clinical trials. Br J Clin Pharmacol. 2020; 86: 687-697. doi: $10.1111 /$ bcp. 14240

4. Breckenridge A, Aronson JK, Blaschke TF, Hartman D, Peck CC, Vrijens B. Poor medication adherence in clinical trials: Consequences and solutions. Nat Rev Drug Discov. 2017; 16(3): 149-150. doi: $10.1038 / \operatorname{nrd} .2017 .1$

5. Murali KM, Mullan J, Chen JH, Roodenrys S, Lonergan M. Medication adherence in randomized controlled trials evaluating cardiovascular or mortality outcomes in dialysis patients: A systematic review. BMC Nephrol. 2017; 18(1): 42. doi: 10.1186/s12882-0170449-1

6. Marrazzo JM, Ramjee G, Richardson BA, Gomez K, Mgodi N, Nair G, et al. Tenofovir-based preexposure prophylaxis for HIV infection among African women. N Engl J Med. 2015; 372(6): 509518. doi: 10.1056/NEJMoa1402269 
7. Applied Clinical Trials. Non-Adherence: A Direct Influence on Clinical Trial Duration and Cost. Website. https://www.appliedclinicaltrialsonline.com/view/non-adherence-direct-influence-clinical-trial-duration-and-cost. 2017. Acce

ssed June 9, 2021.

8. Browne SH, Peloquin C, Santillo F, Haubrich R, Muttera L, Moser $\mathrm{K}$, et al. Digitizing medicines for remote capture of oral medication adherence using co-encapsulation. Clin Pharmacol Ther. 2018; 103(3): 502-510. doi: 10.1002/cpt.760

9. Chai PR, Goodman G, Bustamante MJ, Mohamed Y, Castillo-Mancilla J, Boyer EW, et al. Long-term stability of the electronic sensor component of a digital pill system in real-world storage settings. J Pharm Tech. 2021; 37: 1-5. doi: 10.1177/8755122520985219

10. Plowman RS, Peters-Strickland T, Savage GM. Digital medicines: Clinical review on the safety of tablets with sensors. Expert Opin Drug Saf. 2018; 17(9): 849-852. doi: 10.1080/14740338.2018.1508447

11. Eisenberger U, Wüthrich RP, Bock A, Ambühl P, Steiger J, Intondi A, et al. Medication adherence assessment: High accuracy of the new ingestible sensor system in kidney transplants. Transplantation. 2013; 96: 245-250. doi: 10.1097/TP.0b013e31829b7571

12. Baumgartner S, Buffkin DE Jr, Rukavina E. A novel digital pll system for medication adherence measurement and reporting: A usability validation study. JMIR Preprints. 2021; 30786.

13. Chai PR, Carreiro S, Innes BJ, Rosen RK, O’Cleirigh C, Mayer $\mathrm{KH}$, et al. Digital pills to measure opioid ingestion patterns in emergency department patients with acute fracture pain: A pilot study. J Med Internet Res. 2017; 19(1): e19. doi: 10.2196/jmir.7050

14. Otsuka America Pharmaceutical, Inc., Purchases the Assets of Proteus Digital Health, Inc. Otsuka Website. https://www.otsuka-us.com/discover/proteus-assets-purchase. Accessed June 9, 2021.

15. Abilify Mycite[package insert]. Tokyo, Japan: Otsuka Inc.; 2020. 16. Aldeer M, Martin RP. Medication adherence monitoring using modern technology. Paper presented at: IEEE 8th Annual Ubiquitous Computing, Electronics and Mobile Communication Conference (UEMCON); 2017; 491-497; New York, NY, USA; doi: 10.1109/UEMCON.2017.8249101
17. Shen J, Swift B, Mamelok R, Pine S, Sinclair J, Attar M. Design and conduct considerations for first-in-human trials. Clin Transl Sci. 2019; 12(1): 6-19. doi: 10.1111/cts.12582

18. Detry MA, Lewis RJ. The intention-to-treat principle: How to assess the true effect of choosing a medical treatment. JAMA. 2014; 312(1): 85-86. doi: 10.1001/jama.2014.7523

19. Makubate B, Donnan PT, Dewar JA, Thompson AM, McCowan C. Cohort study of adherence to adjuvant endocrine therapy, breast cancer recurrence and mortality. Br J Cancer. 2013; 108(7): 1515-1524. doi: $10.1038 /$ bjc.2013.116

20. Gater A, Heron L, Abetz-Webb L, Coombs J, Simmons J, Guilhot, et al. Adherence to oral tyrosine kinase inhibitor therapies in chronic myeloid leukemia. Lenk Res. 2012; 36(7): 817-825. doi: 10.1016/j.leukres.2012.01.021

21. McCowan C, Wang S, Thompson AM, Makubate B, Petrie DJ. The value of high adherence to tamoxifen in women with breast cancer: A community-based cohort study. Br J Cancer. 2013; 109(5): 1172-1180. doi: 10.1038/bjc.2013.464

22. Marin D, Bazeos A, Mahon F, Eliasson L, Milojkovic D, Bua $\mathrm{M}$, et al. Adherence is the critical factor for achieving molecular responses in patients with chronic myeloid leukemia who achieve complete cytogenetic responses on imatinib. J Clin Oncol. 2010; 28(14): 2381-2388. doi: 10.1200/JCO.2009.26.3087

23. Burks J, Marshall TS, Ye X. Adherence to disease-modifying therapies and its impact on relapse, health resource utilization, and costs among patients with multiple sclerosis. Clinicoecon Outcomes Res. 2017; 9: 251-260. doi: 10.2147/CEOR.S130334

24. Chai PR, Goodman G, Bustamante M, Mendez L, Mohamed Y, Mayer $\mathrm{KH}$, et al. Design and delivery of real-time adherence data to men who have sex with men using antiretroviral pre-exposure prophylaxis via an ingestible electronic sensor. AIDS Behav. 2021; 25(6): 1661-1674. doi: 10.1007/s10461-020-03082-y

25. Hwang TJ, Carpenter D, Lauffenburger JC, Wang B, Franklin JM, Kesselheim AS. Failure of investigational drugs in latestage clinical development and publication of trial results. JAMA Intern Med. 2016; 176(12): 1826-1833. doi: 10.1001/jamainternmed.2016.6008 